\title{
R.F.-SPUTTERED CO-Cr LAYERS FOR PERPENDICULAR MAGNETIC RECORDING \\ I: STRUCTURAL PROPERTIES
}

J. C. LODDER, T. WIFI INGA AND J. WORST

Department of Electrical Engineering, Twente University of Technology, 7500 AE Enschede (The Netherlands,

(Received July 12,1982; accepted November 9, 1982)

Co-Cr layers for the perpendicular recording mode were deposited by means of r.f. sputtering under suitable conditions in an argon plasma. The films werc characterized structurally by X-ray and electron microscopy and the magnetic properties were determined with a vibrating sample magnetometer and by torque measurements.

The influence of some sputter parameters on the structural and magnetic properties are discussed. Even very thin layers show a high h.c.p. [001] orientation on several substrates. The in-plane magnetic remanence $S_{\mathrm{i}}\left(=M_{\mathrm{r}} / \boldsymbol{M}_{\mathrm{s}}\right)$ is very small (about $4 \%$ ). As will be shown in Part II the magnetic anisotropy is caused by crystal anisotropy. The correlation between the h.c.p. $c$ axis orientation and $S_{\|}$supports this conclusion. The growth mechanism of the layer is discussed.

\section{INTRODUCTION}

Thin ferromagnetic films with perpendicular magnetization have been proposed for high density recording and are considered to be superior to films magnetized in the longitudinal mode ${ }^{1-3}$. Cobalt has a large magnetocrystalline uniaxial anisotropy in the direction of the h.c.p. $c$ axis. In the course of developing a medium with perpendicular magnetic anisotropy several cobalt-metal alloys have been developed ${ }^{4.5}$. Up to now sputtered Co-Cr films have been accepted as the alloy films with the best properties for high density recording. These properties, of course, depend on the r.f. sputtering conditions as has been discussed by several researchers ${ }^{6 * 8}$. New results from standstill recording experiments on our sputtered Co- $\mathrm{Cr}$ films have recently been published".

In this paper (Part I) we describe the structural properties of our films which are made by using optimized sputter parameters with various substrates. The typical development of the structure of the $\mathrm{Co}-\mathrm{Cr}$ layers was studied in detail by X-ray diffraction and $X$-ray rocking curve techniques, special attention being paid to the initial layer ( $25 \mathrm{~nm}$ or less). Furthermore transmission electron microscopy (TEM) and scanning electron microscopy (SEM) techniques were used for the study of both the morphology and the growth processes of the crystallites. 


\section{2. preparation of THE films}

A Leybold-Heraeus $Z 400$ commercial r.f. sputtering system was used. The most important conditions for reproducible deposition of films are listed in Table I. Two types of target were used for producing $\mathrm{Co}-\mathrm{Cr}$ layers, namely a multitarget (cobalt target covered with 13 round chromium pieces) and an alloyed target (Materials Research Company). Using the standard conditions mentioned in Table I we obtained layers with a chemical composition of 15 at. $\% \mathrm{Cr}$ for the multitarget and 19 at. $\% \mathrm{Cr}$ for the alloyed target. The chemical composition and the film thickness were determined by $\mathrm{X}$-ray fluorescence ${ }^{10}$. No significant variation in the chemical composition was measured over an area of $5 \mathrm{~cm}^{2}$ in the centre of the substrate holder. This is reflected by the constant value of the saturation magnetization $M_{\mathrm{s}}$ which is strongly dependent on the composition.

TABLE I

SPUTTER CONDITIONS

\begin{tabular}{ll}
\hline Background pressure & $<10^{-7} \mathrm{mbar}$ \\
Ar pressure & $4 \times 10^{-3} \mathrm{mbar}$ \\
R.f. voltage & $1.6 \mathrm{kV}$ \\
R.f. current & $225 \mathrm{~mA}$ \\
Deposition rate & $2.2 \AA \mathrm{s}$ \\
Target-substrate distance & $50 \mathrm{~mm}$ \\
Target diameter & $100 \mathrm{~mm}$ \\
Substrate holder temperature & Ambient (water cooled) \\
\hline
\end{tabular}

In order to obtain reproducible layers of high quality the following procedure is used.

(i) The bell-jar is baked for $10 \mathrm{~h}$ at $100^{\circ} \mathrm{C}$ until a back pressure of less than $1.5 \times 10^{-7}$ mbar is reached. By means of a liquid $\mathrm{N}_{2}$ Meissner trap the pressure is further decreased to below $10^{-7}$ mbar.

(ii) The target is cleaned before deposition by presputtering ( $15 \mathrm{~min}$ at $1.6 \mathrm{kV})$.

(iii) The substrates are cleaned by glow discharge $(10 \mathrm{~min}$ at $500 \mathrm{~V})$ before deposition.

The substrates are placed on a water-cooled substrate holder. During sputtering the substrate holder attains an equilibrium temperature of $150^{\circ} \mathrm{C}$ after about $1 \mathrm{~h}$. This temperature rise is caused mainly by electron heating from the plasma.

During growth a so-called hot absorption layer is present ${ }^{11}$ just above the condensing surface where the sputter atoms and the electrons from the plasma reach the surface. There is evidence that the surface temperature may be much higher than the measured substrate holder temperature ${ }^{12}$. The growth process is mainly determined by the surface temperature of the layer (at approximately $500^{\circ} \mathrm{C}^{11,13}$ ). Standard size $10 \mathrm{~mm} \times 10 \mathrm{~mm}$ substrates of Corning glass 7059 , silicon wafers $([100]$ oriented) and for TEM examination freshly cleaved mica sheets covered with a thin evaporated amorphous carbon film are used. In order to obtain a good h.c.p. structure with [001] perpendicular to the substrate a very low back pressure (less than $10^{-7}$ mbar) is necessary ${ }^{6-8}$. The argon gas used must be of a very high purity $(99.998 \%)$. 
Impurities cause a decrease in the $c$ axis orientation and are directly noticeable in the magnetic properties, especially $S_{\|}$. Use of the Meissner trap gave $S_{\|}=0.03$ and without the trap we obtained $S_{\|}=0.06$. In addition it has been shown ${ }^{13}$ that a small amount of residual $\mathrm{N}_{2}$ gas in the r.f. sputter chamber promotes the formation of the f.c.c. phase in Co-Cr films which also destroys the well-oriented h.c.p. structure.

\section{INFLUENCE OF THE SUBSTRATE}

During every run we sputtered $\mathrm{Co}-\mathrm{Cr}$ films on silicon, glass and mica covered with carbon. The influence of the different substrates on the $c$ axis orientation is shown in Fig. 1. In this figure $\Delta \theta_{50}$ is given as a function of the film thickness for the multitarget. The difference between glass and silicon is more pronounced for thin layers. This difference may be caused by the effect of electrical charge on the electrically insulating glass substrate from the sputter plasma.

Electrically conductive $\mathrm{C} /$ mica substrates show approximately the same dependence as the silicon substrate although the heat conductivity of $\mathrm{C} / \mathrm{mica}$ is just as low as that of glass. The influence of the substrate is less apparent for thicker layers. These results are supported by the results of $S_{\|}$measurements on the same layers (Fig. 2). From our other magnetic measurements also we can conclude that the properties of $\mathrm{Co}-\mathrm{Cr}$ sputtered on $\mathrm{C} / \mathrm{mica}$ and silicon are practically the same.

It was found that glow discharge cleaning of the substrate is essential in order to obtain a well-oriented layer directly from the start of growth. Measurements of $\Delta \theta_{50}$ for a $\mathrm{Co}-\mathrm{Cr}$ layer $25 \mathrm{~nm}$ thick on a cleaned and an ordinary silicon substrate yielded values of $1.5^{\circ}$ and $5.5^{\circ}$ respectively. With secondary ion mass spectrometry it is clear that the influence of glow discharging is mainly the removal of impurities such as $\mathrm{H}$, $\mathrm{H}_{2} \mathrm{O}$ and $\mathrm{O}_{2}$ from the surface. It is understandable that these impurities influence the growth of the layer. From the litcrature it is known that residual gases can have a strong influence on the crystal habit ${ }^{13}$ and in the case of $\mathrm{Co}-\mathrm{Cr}$ layers an f.c.c. structure ${ }^{14}$ has also been observed.
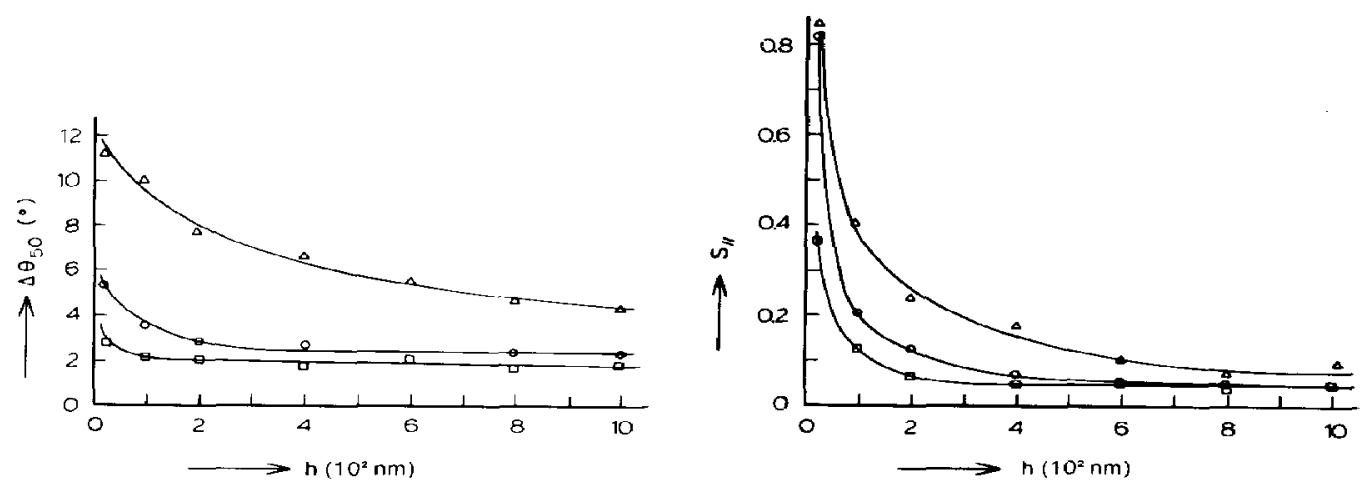

Fig. 1. The relation between the halfwidth angle $\Delta \theta_{50}$ of the $\mathrm{X}$-ray rocking curves and the layer thickness for various substrates: $\triangle$, glass; $\square$, silicon; $0, \mathrm{C} / \mathrm{mica}$.

Fig. 2. The in-plane remanence $S_{\|}$as a function of the film thickness $h$ for various substrates (symbols as in Fig. 1). 


\section{FILM STRUCTURE}

\subsection{X-ray diffraction}

Several Co-Cr layers (15 at. $\%$ Cr) $2 \mu \mathrm{m}$ thick sputtered on $\mathrm{C} / \mathrm{mica}$ were removed from their substrates and powdered in an agate mortar. From this powder an X-ray diffraction spectrum was obtained at $35 \mathrm{kV}$ and $40 \mathrm{~mA}$ with $\mathrm{Cu} \mathrm{K}$ radiation. Only the h.c.p. reflections were present in the $\mathrm{Co}-\mathrm{Cr}$ spectrum measured. The X-ray reflections present are given in Table II. A $\mathrm{KCl}$ powder was used as calibrating material ${ }^{15}$. The lattice parameters $a=2.52 \AA$ and $c=4.06 \AA$ were calculated from the data given in Table II. These give a $c / a$ ratio of 1.62 which is almost the same as the ratio for h.c.p. $\alpha-\mathrm{Co}^{16}$. The $2 \theta$ values for our $\mathrm{Co}-\mathrm{Cr}$ show a small systematic shift with respect to those for $\alpha-$ Co.

TABLE II

MEASURED X-RAY DATA FROM POWDERED SPUTTERED CO-Cr LAYERS COMPARED WITH DATA FOR $\propto$-Co ${ }^{\circ}$

\begin{tabular}{|c|c|c|c|}
\hline \multicolumn{2}{|c|}{ Results for powdered $\mathrm{Co}-\mathrm{Cr}$} & \multicolumn{2}{|c|}{ Data for $\alpha-C o$} \\
\hline Corrected $2 \theta\left(\lambda_{\mathrm{Cu}}\right)(\mathrm{deg})$ & Calculated d $(\AA)$ & $d(\AA)$ & $h k i l$ \\
\hline 41.46 & 2.175 & 2.165 & 1010 \\
\hline 44.65 & 2.030 & 2.023 & 0002 \\
\hline 47.36 & 1.919 & 1.910 & $10 \overline{1}$ \\
\hline 62.53 & 1.485 & 1.48 & $10 \overline{12}$ \\
\hline 75.58 & 1.258 & 1.252 & $11 \overline{20}$ \\
\hline \multirow[t]{2}{*}{84.19} & 1.150 & 1.149 & $10 \overline{13}$ \\
\hline & & 1.083 & $20 \overline{20}$ \\
\hline 92.23 & 1.069 & 1.066 & $11 \overline{2} 2$ \\
\hline 94.18 & 1.052 & 1.047 & 2021 \\
\hline 98.78 & 1.015 & 1.015 & 0004 \\
\hline
\end{tabular}

From ref. 16.

\subsection{Rocking curve method}

The preferred crystallographic orientation (texture) was examined by measuring the layers with a Philips texture goniometer using Co K $\alpha$ radiation. The $\{0002\}$ and $\{1011\}$ pole distributions were measured with a pitch of $2.5^{\circ}$ by means of the Schultz method ${ }^{17}$. The $\{0002\}$ pole figure shows only a very narrow distribution in the centre of the plot and that for $\{10 \overline{1} 1\}$ (Fig. 3) consists of a narrow circular distribution which can be assigned to the $[001] 1^{\circ}$ orientation. Because these pole figures showed a high degree of rotational symmetry we decided to examine all other layers by the so-called rocking curve method where the specimen is scanned in a curved line instead of spirally as in the Schultz method. In Fig. 4 the $\{0002\}$ rocking curves are given for the initial layer (Fig. 4(a)) and for thick layers sputtered on silicon (Fig. 4(b)) and glass (Fig. 4(c)). From these curves the halfwidth angle $\Delta \theta_{50}$ was determined. This angle represents the dispersion of the [001] direction ( $c$ axis) of the h.c.p. structure around the normal to the film plane.

$\Delta \theta_{50}$. for very thin layers $(25 \mathrm{~nm})$ is small $\left(3^{\circ}\right)$ when the layers have been sputtered on silicon substrates (Fig. 4(a)). This means that the initial growth of the $c$ axis is already highly oriented. In other words the initial layer grows with the $\{0002\}$ plane parallel to the substrate. The relation between $\Delta \theta_{50}$ and the film thickness for 
various substrates is given in Fig. 1. From this it can be seen that $\Delta \theta_{50}$ improves during growth of the film. For a thickness of more than $0.1 \mu \mathrm{m}$ the $c$ axis orientation for $\mathrm{Co}-\mathrm{Cr}$ films on silicon is almost constant $\left(1.7^{\circ}\right)$. Films sputtered on $\mathrm{C} / \mathrm{mica}$ show a similar behaviour, but those on glass have a much worse orientation at the start and $\Delta \theta_{50}$ is not constant for thicknesses of up to $1.5 \mu \mathrm{m}$.

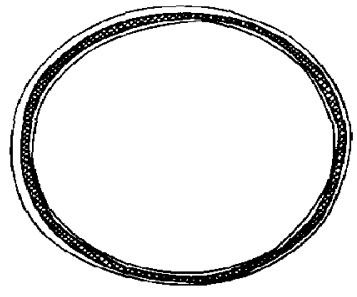

I

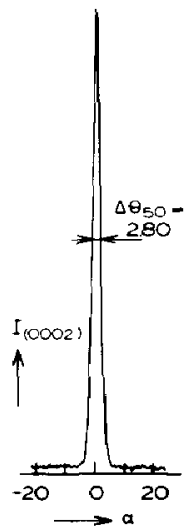

(a)

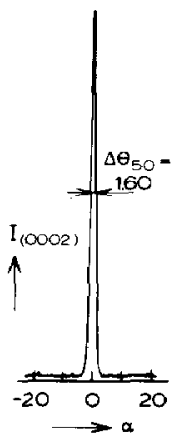

(b)

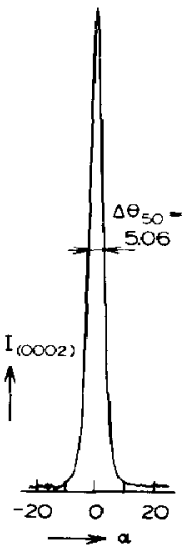

(c)

Fig. 3. The $\{10 \overline{1} 1\}$ pole figure for $\mathrm{Co}-\mathrm{Cr}$ film (15 at \% $\mathrm{Cr}$ ) $800 \mathrm{~nm}$ thick sputtered on silicon: $\square .2 \%$; $30 \%$. The centre of the plot indicates the film normal.

Fig. 4. The $\{0002\} X$-ray rocking curves for sputtered Co-Cr layers: (a) on silicon $\left(25 \mathrm{~nm} ; \Delta \theta_{\mathrm{so}}=2.8^{\prime}\right)$; (b) on silicon $\left(800 \mathrm{~nm} ; \Delta \theta_{50}=1.6^{\circ}\right) ;(\mathrm{c})$ on glass $\left(800 \mathrm{~nm} ; \Delta \theta_{50}=5.06\right)$.

Other experiments were carried out to investigate whether the initial layer always starts growing with the basal $\{0002\}$ h.c.p. planes parallel to the substrate. A silicon substrate was placed at an angle of $45^{\circ}$ to the substrate holder next to a normally positioned substrate. There was only a slight difference between the two sputtered layers of thickness $25 \mathrm{~nm}$. The dispersion of the $c$ axis orientation was $5.5^{\circ}$ and $3.0^{\circ}$ respectively. This definitely shows that for sputtered $\mathrm{Co}-\mathrm{Cr}$ layers the $\{0002\}$ h.c.p. plane grows parallel to the substrate.

\subsection{Electron microscopy}

The crystal diameter was determined by means of electron microscopy. Relatively thin films up to $200 \mathrm{~nm}$ were examined with a JEOL $200 \mathrm{CX}$ microscope $(200 \mathrm{kV})$. Films of thickness up to approximately $600 \mathrm{~nm}$ were studied with the high voltage (1000 kV) TNO electron microscope. (This instrument, designed and constructed at the Institute of Applied Physics TNO-TH Delft, has been installed at the Metal Research Institute TNO, Apeldoorn.)

The crystal diameter on the surface was determined by a replica technique as well as from images made with the JEOL $200 \mathrm{CX}$ microscope in the SEM mode. The films measured by transmission microscopy were sputtered on freshly cleaved mica substrates covered with a thin amorphous evaporated layer of carbon (thickness, approximately $30 \mathrm{~nm}$ ). After the layer had been cut into pieces they were dipped in water for separation from the mica and mounted on grids. In Fig. 5 the bright field and electron diffraction images from the initial layer and relatively thick layers 

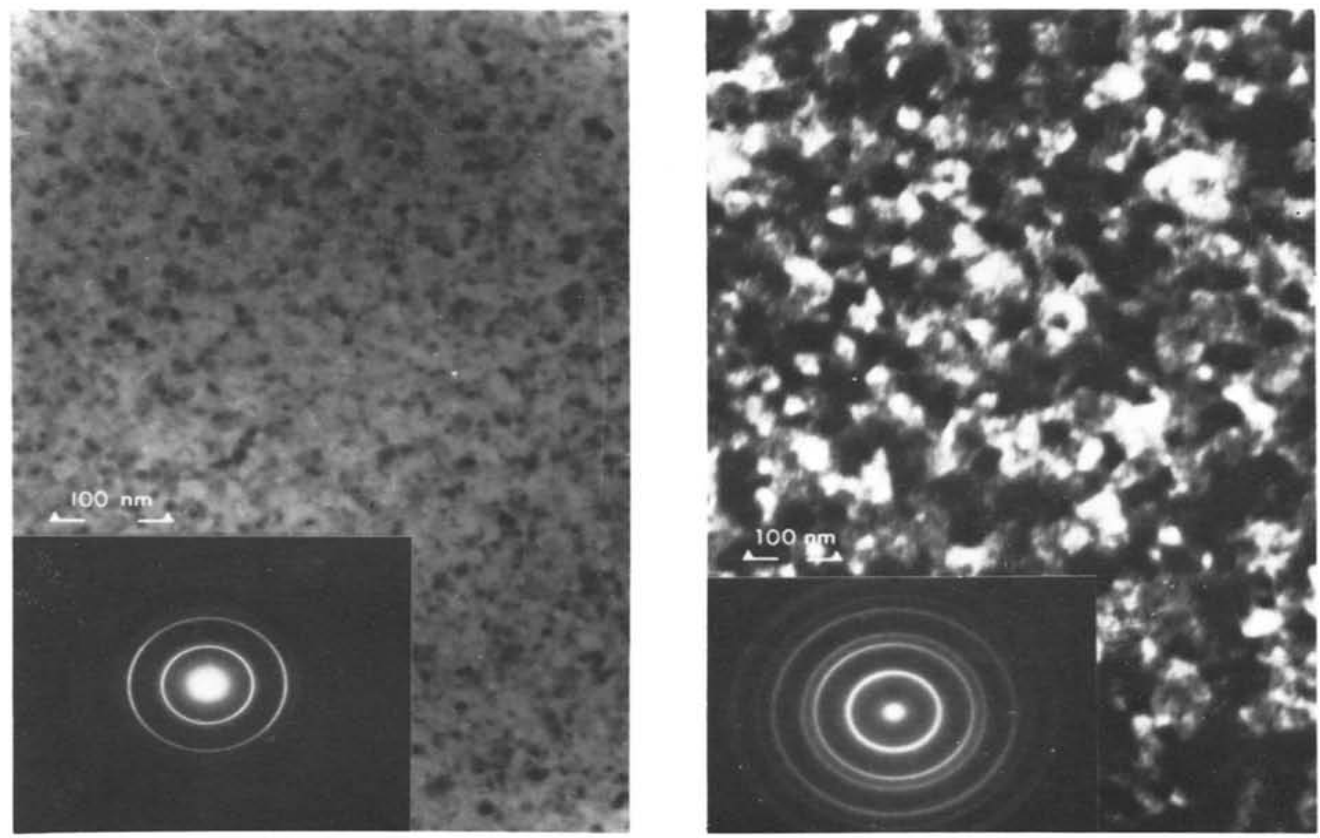

(a)

(b)
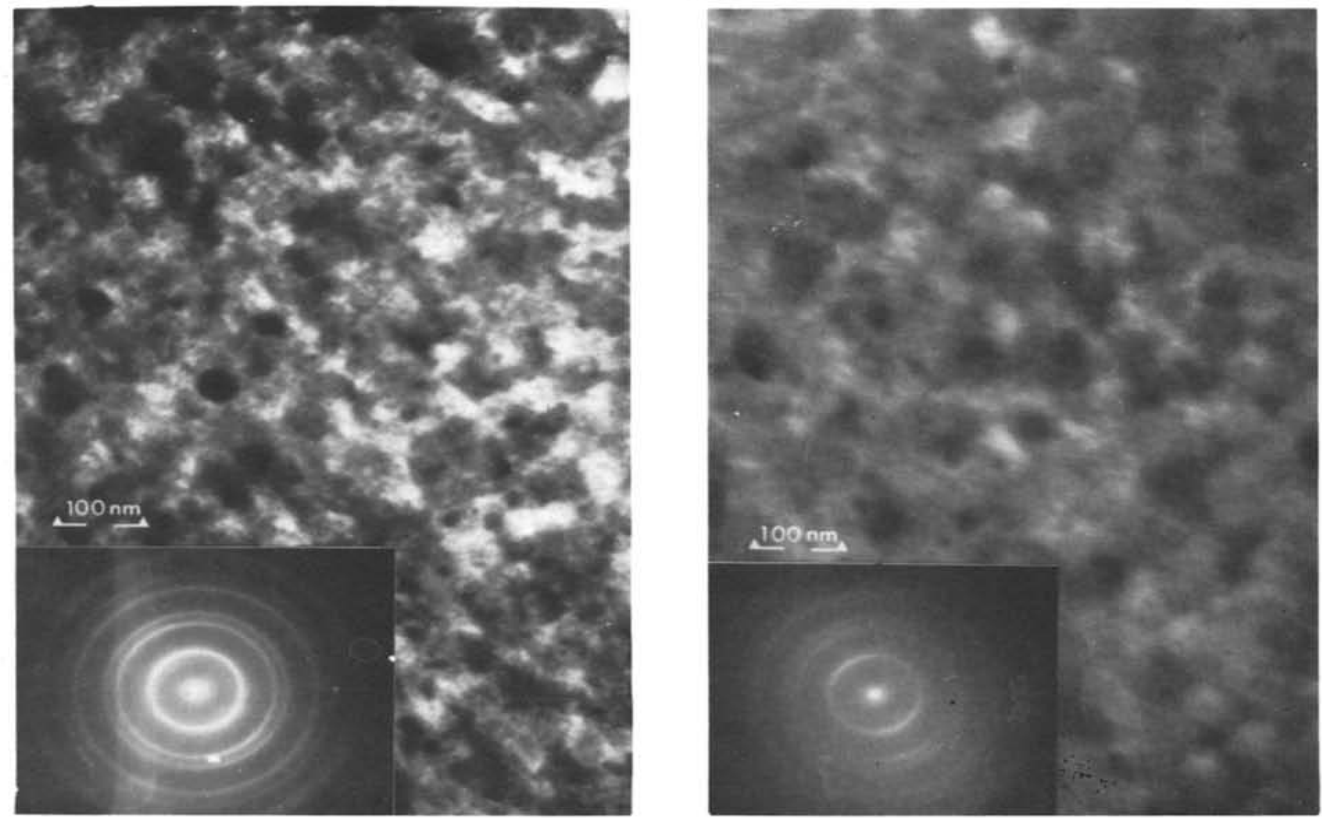

(c)

(d)

Fig. 5. Transmission electron micrographs (high voltage electron microscope) and the corresponding diffraction patterns for sputtered Co-Cr layers of various thicknesses: (a) $25 \mathrm{~nm}$; (b) $200 \mathrm{~nm}$; (c) $400 \mathrm{~nm}$; (d) $600 \mathrm{~nm}$. 
are shown. From the diffraction patterns it can be seen that only h.c.p. reflections were present.

The crystal diameter was determined as a function of the layer thickness by microscopic examination. The crystal diameter appears to increase with the film thickness (Fig. 6). A two-stage replica technique for imaging the surface structure of $\mathrm{Co}-\mathrm{Cr}$ layers was used for very thick layers (above $0.5 \mu \mathrm{m}$ ). First a negative replica is made with Technovite (type 4071) and from this the final positive replica is prepared using carbon and platinum shadowing. After the Technovite has been dissolved the final replica can be studied. A typical electron micrograph is shown in Fig. 7.

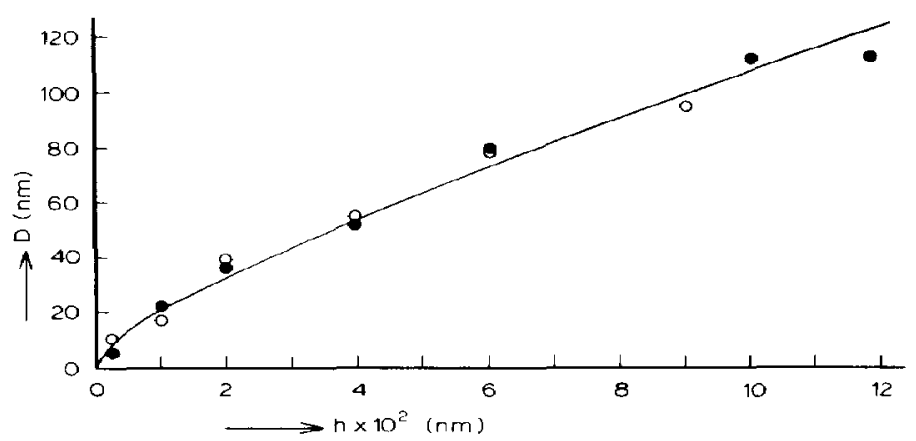

Fig. 6. The crystal size $D$ as a function of the layer thickness $h: 0$, multitarget; $\bigcirc$, alloyed target.

Cross-sectional structures.can be observed with the JEOL 200 CX microscope in the SEM mode. These samples are prepared by cooling the specimen in liquid $\mathrm{N}_{2}$ before breaking it to obtain a brittle fracture. Figure 8 shows the cross-sectional structure of a Co-Cr film $2 \mu \mathrm{m}$ thick sputtered on silicon. Such micrographs do not show a columnar-like morphology as can mainly be seen in other, relatively thick, sputtered layers (see for instance ref. 18). From these and the TEM examination we concluded that the crystals tend to be conically shaped (Section 5).

\section{DISCUSSION OF THE FILM MORPHOLOGY AND STRUCTURE}

The film structure is usually described by its two most important properties: the crystallographic (preferred) orientation (or texture) and the morphology (crystal size and shape). These two features of thin films are often described independently ${ }^{18-21}$ but sometimes there is a strong relation between them. Especially in the case of the "survival of the fastest" model 19 the morphology is strongly determined by the crystallographic orientation; i.e. the film orientation is finally given by the crystallographic axis which grows the fastest. In the h.c.p. structure this is the [001] axis.

With physical vapour deposition techniques these properties are more or less determined by the process involved, i.e. in sputtering by the process parameters: sputter rate, substrate temperature, contamination and argon pressure. Here it is assumed that the argon pressure used does not influence the film properties. At higher pressures the $c$ axis orientation is disturbed ${ }^{8}$. It is also assumed that the rate 


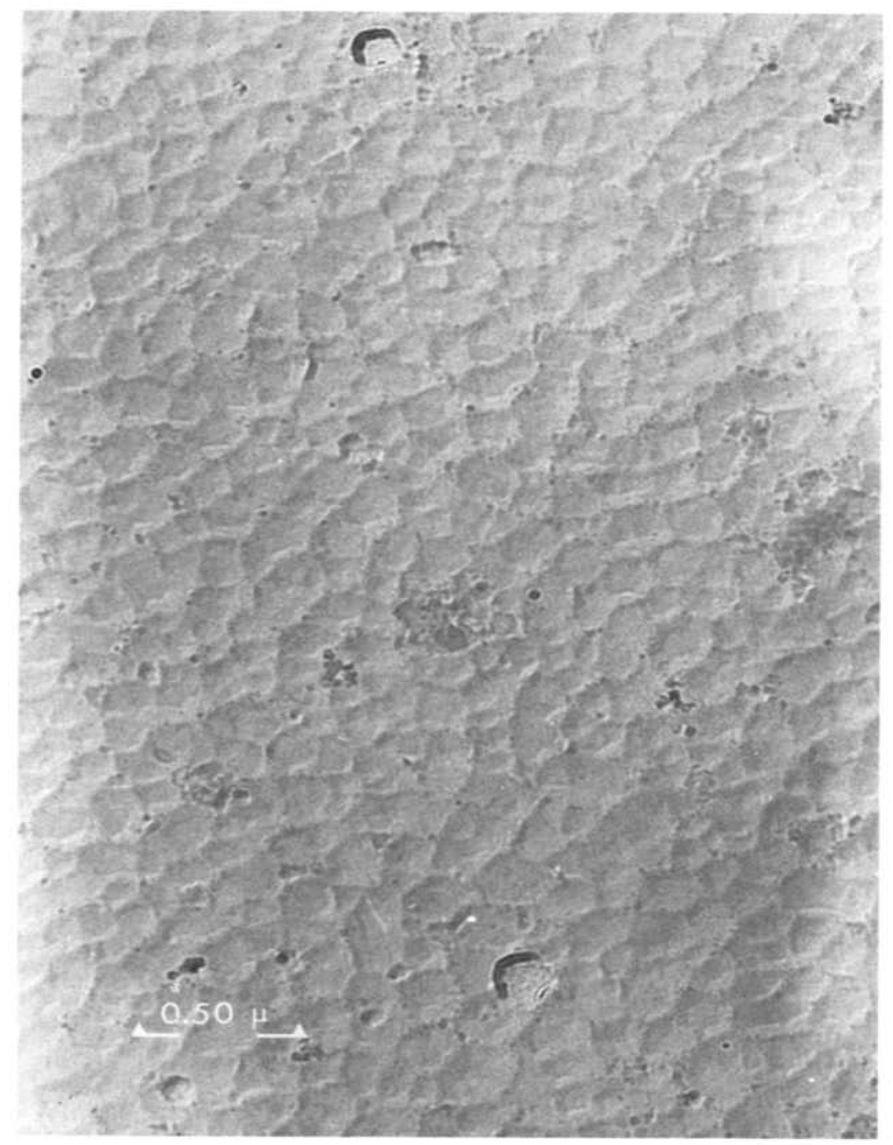

Fig. 7. An indirect replica of a $\mathrm{C}$ o-Cr layer $1 \mu \mathrm{m}$ thick.

of deposition by evaporation ${ }^{22}$ or by several sputtering methods ${ }^{6 \cdot 8.23}$ does not effect the perpendicular $c$ axis orientation. From this it can be seen that a perpendicular $c$ axis orientation is obtained at rates between 0.2 and $600 \mathrm{~nm} \mathrm{~s}{ }^{-1}$. It is not yet clear whether the deposition rate influences the film morphology of $\mathrm{Co}-\mathrm{Cr}$ films. However, it has been shown ${ }^{18}$ that the morphology of one-phase layers does not change in a wide range of deposition rates.

The most important parameter, but unfortunately also the most elusive parameter in the case of sputtering, is the temperature. In r.f. sputtering, the surface temperature, i.e. at which the film formation takes place, is predominantly determined by three factors which are of the same order of magnitude: (i) the temperature of the substrate holder, (ii) the temperature produced by heat from the condensing atoms and (iii) the temperature produced by heating from the sputter plasma, mainly by the electrons. This complex heat mechanism not only makes it difficult to determine an accurate surface temperature (whatever this may mean) but also implies that this temperature will not be stationary during the sputter run. The 
surface temperature in r.f. sputtering was determined by measuring the h.c.p.-f.c.c. transition temperature and comparing this with bulk data for $\mathrm{Co}-\mathrm{Cr}$ and was found to be $550^{\circ} \mathrm{C}$ higher than the measured substrate temperature ${ }^{12}$. However, surface formation should not be compared directly with bulk formation. Besides the unknown deposition temperature there is also some ambiguity in the literature about the dependence of the $c$ axis orientation on the substrate temperature ${ }^{7.8}$.

Despite this uncertainty about the temperature the overall picture of our films is quite consistent and shows clear features of the film growth. First, the $c$ axis

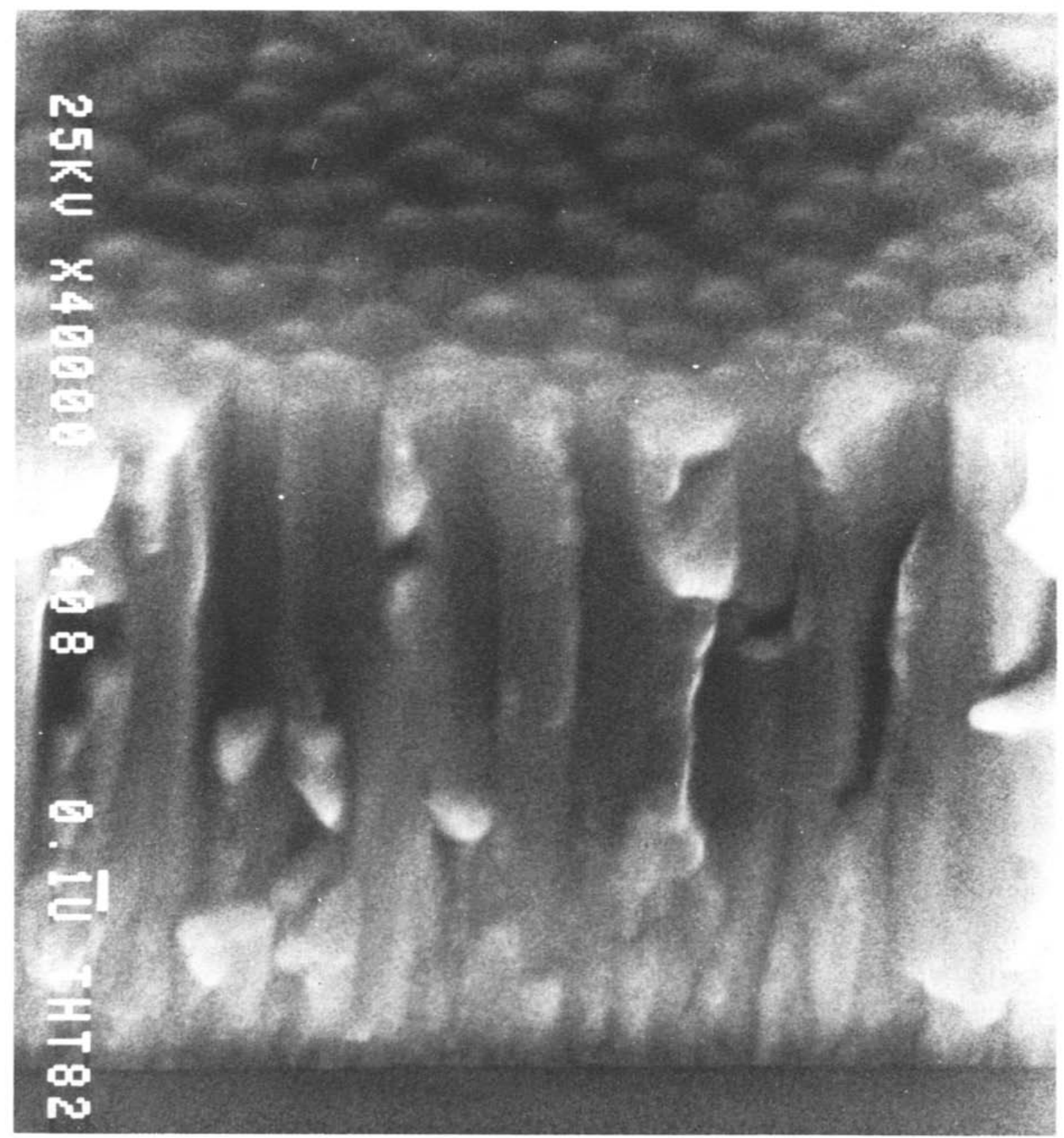

Fig. 8. Cross-sectional SEM image of a Co-Cr layer $2 \mu \mathrm{m}$ thick sputtered on silicon 
orientation is excellent, being independent of the film thickness from very thin films up to a film thickness of a few microns. Secondly, the grain size increases continuously with the film thickness as measured for films in the range $25-2000 \mathrm{~nm}$. After $1 \mathrm{~h}$ a constant substrate temperature of $150^{\circ} \mathrm{C}$ is reached with a sputter rate of $0.2 \mathrm{~nm} \mathrm{~s}^{-1}$. The thickness range examined far exceeds the value measured when the temperature became stationary. Therefore it is assumed that during film growth the surface temperature can be considered as constant; i.e. at least temperature variations are too small to cause different growth characteristics. The relative surface temperature (relative to the melting point) was evaluated as about $0.3-0.5$.

The excellent $c$ axis orientation of the nuclei probably originates from the tendency to condense with the most densely populated crystal planes, the $\{0002\}$ planes, parallel to the substrate. This is supported by the excellent $c$ axis orientation $\left(\Delta \theta_{50}=5^{\circ}\right)$ of a $\mathrm{Co}-\mathrm{Cr}$ film $25 \mathrm{~nm}$ thick, sputtered on a silicon substrate which was placed at an angle of $45^{\circ}$ to the substrate holder.

The preferred orientation is maintained as the film thickness is increased. This could be caused by a mechanism of self-epitaxy. In this case, however, the orientation would deteriorate with increasing film thickness ${ }^{19}$. It is more likely that this normal $c$ axis orientation is maintained because it is also the fastest growing crystallographic orientation. It has been shown ${ }^{19}$ that a certain crystallographic orientation can arise from a mechanism described as "the survival of the fastest", i.e. the crystallites with the fastest growing axis (perpendicular to the substrate) survive, suppressing crystallites with other orientations. This type of layer is therefore recognizable by a poorly oriented initial layer, as is the case when glass substrates are used for $\mathrm{Co}-\mathrm{Cr}$ (see Fig. 1). However, when silicon or $\mathrm{C} / \mathrm{mica}$ substrates are used the first nuclei are immediately properly oriented which means that the film grows along its fastest direction from the very beginning.

Now the question remains: Why does the crystal diameter increase with the film thickness? In the case of the "survival of the fastest" there is a simple mechanism which explains this feature. In our case there has to be a different cause. A formal reason could be the crystal boundary energy; i.e. by increasing the crystal diameter the energy density is decreased. This, however, does not explain how some crystals grow at the expense of others.

From our SEM and replica micrographs it can be concluded that there is a large statistical variation in the crystal diameter. Furthermore the crystal boundaries do not show any intermediate layer or separation, the crystallites being very close. A simple explanation could therefore be that the average increase in the crystal diameter is just governed by statistical rules. The relative surface temperature, of the order of 0.4 , is in the range where surface diffusion is believed to be the dominant factor ${ }^{18}$. Therefore a statistical variation in the local flux at the individual crystallites may result in the increase of the "highest" at the expense of the "lowest" crystals which is made possible by the surface diffusion; i.e. the crystallites are capable of growing sideways unhindered by intermediate crystallite layers. Clearly this effect is self-enhancing.

\section{RELATION TO THE MAGNETIC PROPERTIES}

Perpendicular magnetic recording necessitates a perpendicular magnetic 
anisotropy of the layers. Our Co-Cr films had a large magnetocrystalline anisotropy with the $c$ axis of the h.c.p. structure oriented perpendicularly to the film. We measured $M_{\mathrm{s}}$ and the uniaxial magnetic anisotropy constants $K_{1}$ and $K_{2}$ with a vibrating sample magnetometer (VSM) and a torque meter respectively (see Part II). The in-plane and perpendicular $M-H$ loops measured with the VSM are given in Fig. 9. Owing to the excellent preferred $c$ axis orientation the in-plane hysteresis loop is marked by a small in-plane remanence ratio $S_{\|}=0.04$ and a strong anisotropy field $H_{\mathrm{k}}$. This field depends on the film composition and varied for our films between 3 and $7 \mathrm{kOe}$.

The relation between the in-plane remanence and the film thickness for three different types of substrate is given in Fig. 2. In particular the initial layer sputtered on silicon shows a small in-plane remanence caused by the perpendicular orientation of the anisotropy axis. Films sputtered on $\mathrm{C} / \mathrm{mica}$ exhibit approximately the same behaviour as those deposited onto silicon. The minimum in-plane remanence is obtained for films sputtered on silicon to a thickness of $300 \mathrm{~nm}$ or more, while for $\mathrm{C} /$ mica substrates the optimum film thickness is $600 \mathrm{~nm}$ or more. Above these thicknesses there is no change observed up to $2 \mu \mathrm{m}$. Using a glass substrate the best remanence is achieved at a thick ness of approximately $800 \mathrm{~nm}$. We have already shown by $\mathrm{X}$-ray diffraction that the quality of $c$ axis orientation $\left(\Delta \theta_{50}\right)$ was extremely good even for thin layers (see Fig. 1). In Fig. 10 the in-plane remanence is given as a function of the dispersion $\Delta \theta_{50}$ of the h.c.p. $c$ axis orientation. The data plotted in this figure correspond to films which were sputtered at various values of parameters such as the thickness, argon pressure and sputter voltages. The correlation between $\Delta \theta_{50}$ and $S_{\|}$is manifest and this supports the

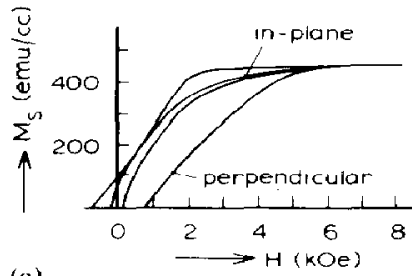

(a)

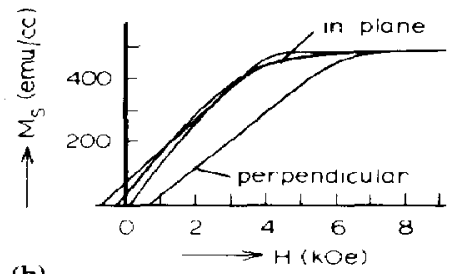

(b)

Fig. 9. $M-H$ loops measured with the VSM for layers (a) $25 \mathrm{~nm}$ and (b) $600 \mathrm{~nm}$ thick sputtered on silicon.

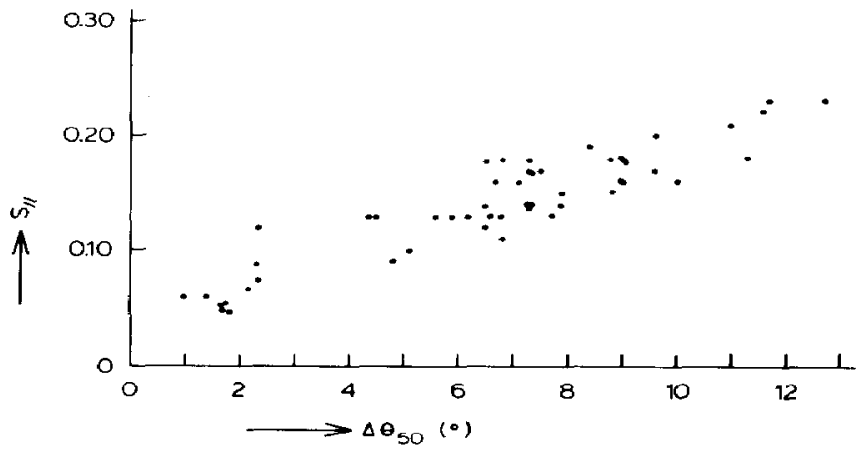

Fig. 10. The in-plane remanence $S_{\|}$as a function of the $c$ axis dispersion $\Delta \theta_{50}$. 
conclusion drawn from our torque measurements (see Part II) that the anisotropy of Co-Cr films originates from crystal anisotropy.

\section{CONCLUSIONS}

Independently of the layer thickness our sputtered Co-Cr layers on silicon substrates have an h.c.p. structure with the [001] $c$ axis perpendicular to the substrate. In order to start directly with a correct orientation it is essential to clean the substrates by the glow discharge method.

The $c / a$ ratio calculated from $X$-ray data is 1.62 . This is almost the same as the ratio for h.c.p. $\alpha-\mathrm{Co}$. The type of substrate influences the growth of the initial layer. The growth model for our layers is based on the tendency of the atoms in $\mathrm{Co}-\mathrm{Cr}$ to condense preferentially with the most densely packed crystal plane, the $\{0002\}$ plane, parallel to the substrate, which also corresponds to the direction of fastest growth.

The formation of the layer takes place in the range where surface diffusion is the dominant factor. The crystal size increases almost linearly with the film thickness. We propose the mechanism for crystal growth to be a statistical variation in the local flux at the individual crystallites, but it may also be influenced by a lowering of the grain boundary energy.

The morphology of our layers shows that they consist of conically shaped crystals. The magnetic anisotropy axis coincides with the $c$ axis of the crystals.

\section{ACKNOWLEDGMENTS}

We should like to express our appreciation to Professor Dr. G. H. Jonker and Professor Dr. J. H. J. Fluitman for their stimulating discussions.

\section{REFERENCES}

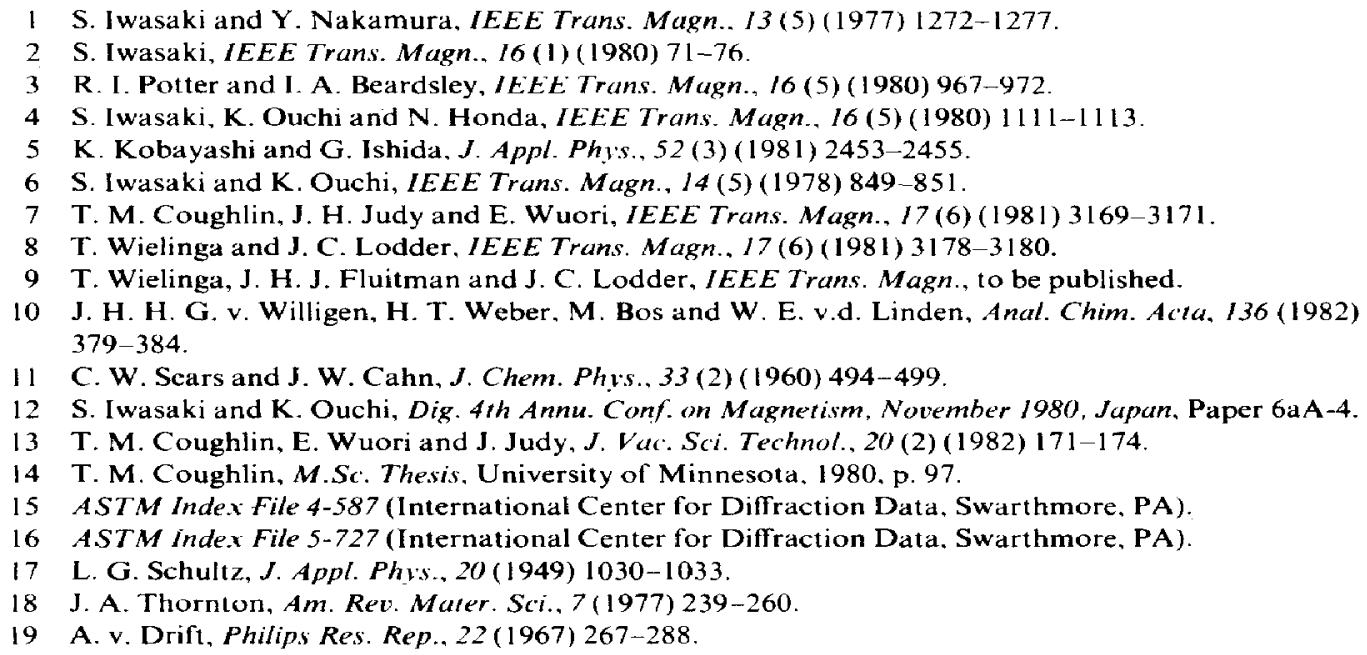


20 E. Bauer, in M. H. Francombe and H. Sato (eds.), Single-Crystal Films, Pergamon, Oxford, 1964, pp. 43-67.

21 V. Sanders, in J. R. Anderson (ed.), Chemisorption and Reaction on Metallic Films, Academic Press, London, 1971, pp. 1-38.

22 R. Sugita, T. Kunieda and F. Kobayashi, IEEE Trans. Magn., 17 (6) (1981) 3172-3174.

23 S. Kadokura, T. Tomie and M. Naoe, IEEE Trans. Magn., 17 (6) (1981) 3175-3177. 